
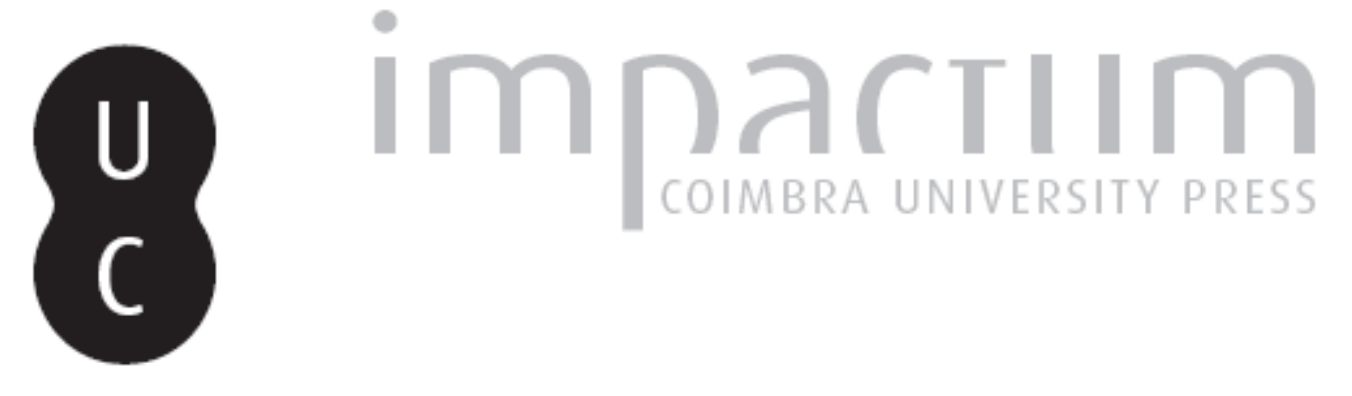

\title{
Genova per loro...: o il filo del discorso fra Antonio Tabucchi e Fernando Lopes
}

Autor(es): $\quad$ Sacco, Marcello

Publicado por: Imprensa da Universidade de Coimbra

URL persistente:

URI:http://hdl.handle.net/10316.2/42609

DOI:

DOl:https://doi.org/10.14195/0870-8584_3_6

Accessed : $\quad$ 26-Apr-2023 15:43:36

A navegação consulta e descarregamento dos títulos inseridos nas Bibliotecas Digitais UC Digitalis, UC Pombalina e UC Impactum, pressupõem a aceitação plena e sem reservas dos Termos e Condições de Uso destas Bibliotecas Digitais, disponíveis em https://digitalis.uc.pt/pt-pt/termos.

Conforme exposto nos referidos Termos e Condições de Uso, o descarregamento de títulos de acesso restrito requer uma licença válida de autorização devendo o utilizador aceder ao(s) documento(s) a partir de um endereço de IP da instituição detentora da supramencionada licença.

Ao utilizador é apenas permitido o descarregamento para uso pessoal, pelo que o emprego do(s) título(s) descarregado(s) para outro fim, designadamente comercial, carece de autorização do respetivo autor ou editor da obra.

Na medida em que todas as obras da UC Digitalis se encontram protegidas pelo Código do Direito de Autor e Direitos Conexos e demais legislação aplicável, toda a cópia, parcial ou total, deste documento, nos casos em que é legalmente admitida, deverá conter ou fazer-se acompanhar por este aviso.

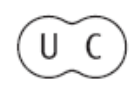




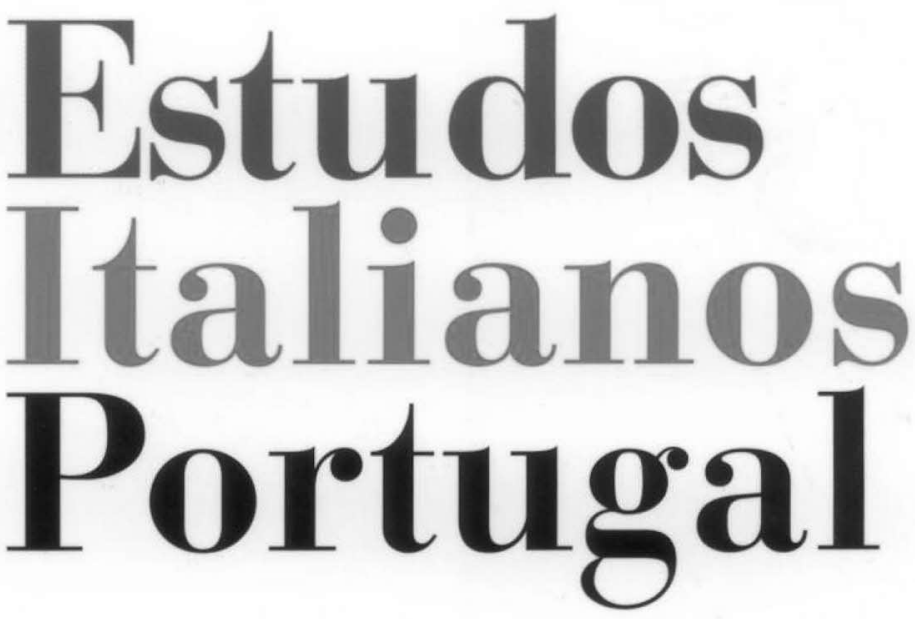

Instituto

Italiano

de Cultura

de Lisboa

Nova Série

$\mathrm{N}^{\circ} 3$

2008 


\section{GENOVA PER LORO ... O IL FILO DEL DISCORSO FRA ANTONIO TABUCCHI E FERNANDO LOPES}

Marcello Sacco ${ }^{\star}$

Per chi facesse attenzione ai dati e date delle filmografie più che a quelli dell'anagrafe non dovrebbe essere difficile individuare in Fernando Lopes l'iniziatore di una certa tendenza del cinema portoghese che avrebbe poi trovato più celebri e prolifici rappresentanti in autori di generazioni lontane, anche precedenti alla sua. Una tendenza, per dirla con Alberto Seixas Santos, che mira alla critica della rappresentazione, alla de-drammatizzazione del dramma, insomma alla fuga dalla tradizione naturalistica con tutto il suo portato di mimetismo, verosimiglianza e trasparenza ${ }^{1}$. Messe le cose in questi termini, potrebbe trattarsi anche della descrizione del lavoro teatrale e multimediale di Carmelo Bene o della coppia Straub/Huillet. Tuttavia sembrerebbe che solo in Portogallo queste istanze siano diventate "tendenza", sorta

* Laurea in Lingue e Letterature Straniere presso l'Università di Lecce e Master in "Estudos Portugueses" presso la Universidade Autónoma di Lisbona, è docente di Italiano presso i corsi di Canto della Academia de Amadores de Música e del Conservatorio di Lisbona. Ha curato e tradotto diverse opere di autori portoghesi, fra cui Almeida Faria, Agustina Bessa-Luís, José Gil, oltre all'antologia, in collaborazione con G. Miraglia, L'Anima Navigante: racconti dal Portogallo (Besa, 2006). Come giornalista, si è occupato per anni di cinema e di teatro.

1 A. Seixas Santos, "Una certa tendenza del cinema portoghese", in R. Turigliatto, S. Fina (ed.), Amori di perdizione. Storie di cinema portoghese 1970-1999, Torino, Lindau, 1999, p. 61. 
di mainstream nazionale (sia pur nell'ambito di una ridottissima produzione cinematografica annuale) di un'estetica che in altri paesi, variamente etichettata, rimaneva, più o meno volutamente, ai margini. Non a caso il titolo di Seixas Santos riecheggia il polemico articolo di Truffaut del '54 che, come sappiamo, si riferiva al cinema francese e a ben altre tendenze. In quell'articolo si parlava anche di "cette fameuse fidélité...", in riferimento agli adattamenti di testi letterari ad opera di sceneggiatori dalla personalità multipla, come Aurenche e Bost, i quali lavoravano indefessi su Gide e Radiguet, Colette e Bernanos, rivelando una certa (sono sempre parole di Truffaut) "souplesse d'esprit".

Nel cinema portoghese la critica della rappresentazione comincia con due opere altrettanto eclettiche, in quanto, a firma dello stesso autore, introducono la nouvelle vague in Portogallo seguendo strade piuttosto diverse. Punto d'incontro (immaginativo più che immaginario) di queste linee quasi parallele è il loro regista, un Fernando Lopes a quel tempo agli esordi, dotato di un'ispirazione magari eteroclita e tuttavia mai più eguagliata. In Belarmino (1964) racconta la storia di un pugile nei modi documentaristici del cinéma-verité, forse credendo ancora in qualche possibilità di afferrare la realtà per la coda sfuggendo alla sintassi narrativa tradizionale (e quale piccola, minuziosa realtà sociale: il microcosmo del pugilato in un quartiere popolare di Lisbona). E invece, qualche anno più tardi, con la virata estetica di Un'ape sotto la pioggia (Uma abelha na chuva, 1972, ma le riprese erano iniziate già nel '68), Lopes rinuncia ad immergersi in una tranche di vita reale per buttarsi in un romanzo e farlo amorevolmente, e letteralmente, a pezzi. Non come ogni sceneggiatura che, operando dei tagli ellittici, smembra e ri-membra (nel senso anche di ricordare a chi lo ha già letto) quel che l'adattatore considera essenziale nel libro, ma piuttosto lasciando i suoi frammenti a vista, disadattati. Dalla tranche de vie alle tranches de livre. Pur ispirandosi a uno dei più bei 
romanzi del secondo Novecento portoghese (di Carlos de Oliveira), l'omonima pellicola si basa su due idee guida: montaggio come contestazione e decostruzione per giungere a un film da non intendersi mai come ipostasi di un'opera letteraria (sia essa un libro preesistente o la stessa sceneggiatura); scollamento fra banda visiva e banda sonora.

Una certa idea di squisitezza letteraria del moderno cinema lusitano discende da quell'esperienza/esperimento (in portoghese è la stessa parola) e passa poi per alcuni dei più significativi film di Manoel de Oliveira. Un cinema tanto più letterario in quanto, contestato il libro in fase di montaggio, caccia la letteratura dalla finestra, ma la fa rientrare dalla porta, ossia con tutti gli onori. I brani letterari letti, scollati e/o diversamente incastonati, assumono l'inedita e inaudita importanza che ha la musica nel film su Bach della coppia Straub/Huillet, mai mera tappezzeria. Se nelle simpatie dei teorici francesi, come Bazin, sul montaggio eisensteiniano doveva prevalere il piano-sequenza per quella sua capacità di captare e trasmettere con l'inquadratura la durata vissuta (durée vecue), il cinema portoghese si inoltra ad esplorare la durée lu, la durata della lettura, che poi troverà in Amor de perdição di Manoel de Oliveira (adattamento di un classico dell'800) il suo azzardo più estremo e avventuroso ${ }^{2}$. Per concludere ancora con le parole di Seixas Santos: "Alla lettura di un'avventura si sostituisce l'avventura di una lettura"3.

Con gli anni '80, anche il cinema portoghese riscopre (e sembra quasi una scoperta adamitica) una certa vena neonarrativa. Fernando Lopes, la cui attività è stata sempre caratterizzata da lunghi periodi di silenzio, non è più un capofila. Dopo l'incursione avanguardistica è rientrato nei

2 "Un film di circa quattro ore che segue il romanzo [...] quasi parola per parola e segna una data storica nei rapporti tra cinema e letteratura", João Bénard da Costa, Cinema portoghese, in Storia del cinema mondiale. L'Europa, a cura di G.P. Brunetta, vol. III, t. II, Torino, Einaudi, 2000, p. 1446.

3 A. Seixas Santos, cit. 
ranghi, ma segue la sua strada concedendosi escursioni in vari ambiti letterari. All'inizio degli anni '90 questa strada incrocia l'orizzonte di Antonio Tabucchi. Il regista forse non si porta più dentro né mire de-strutturalistiche né angosce edipiche, come ai tempi in cui frequentava Carlos de Oliveira (nel frattempo scomparso), ma conserva ancora una pratica da uomo di cinema con la quale abbordare opere appartenenti a una sintassi diversa dalla sua. Se già durante la travagliata lavorazione di Un'ape sotto la pioggia teneva in mente l'opera in versi di Oliveira come punto di fuga e di rottura rispetto al romanzo ${ }^{4}$ a cui il film, come un figlio al padre, doveva il nome, ci sarà anche per Il filo dell'orizzonte un testo occulto che orienta (o magari "disorienta") il lavoro sul testo palese? L'adattamento di un testo si basa sulla trovata di un sottotesto; termine ben noto, da Stanislavski in poi, alla letteratura sulle pratiche attoriali e spettacolari in genere. Sappiamo che il sottotesto ha a che vedere con la ricerca di una motivazione, necessaria per riuscire tutte le sere a piangere puntualmente per Ecuba (dilemma amletico al quale Tabucchi dedica una citazione chiave nelle pagine del libro). Per l'attore è una tecnica, un processo creativo che deve rimanere segreto, privato. Trasposto in ambiti diversi, potremmo tutto sommato dire che il sottotesto è un altro nome per indicare quella pratica che la teoria della letteratura chiama intertestuale, citazionistica o di scrittura a palinsesto. Il risultato, nel nostro caso, è che il romanzo più "lynchano" di Tabucchi (per come decostruisce il giallo tradizionale ripercorrendone i luoghi e le atmosfere senza mai saldarli nella logica ferrea della detection classica) nelle mani di Lopes diventa un film che chiude l'arco narrativo con una circolarità fin troppo perfetta, con quell'identità dichiarata tra vittima e investigatore che ricorda un precedente romanzo tabucchiano, Notturno indiano, dove era effettivamente pos-

\footnotetext{
${ }^{4}$ F. Lopes, "Do rigoroso trabalho oficinal", in Relâmpago, 11, 2002, p. 142.
} 
sibile pensare l'identità degli attanti, protagonista/io-narrante e personaggio da egli ricercato, come se tutto il libro fosse originato dal racconto en abyme fatto alla ragazza che nel capitolo finale siede col narratore al tavolo di un ristorante. Ma nel Filo dell'orizzonte il finale è proprio come l'orizzonte: "un luogo geometrico, perché si sposta mentre noi ci spostiamo", perciò sfocia nella "lost higway" di un appuntamento al buio che non conduce da nessuna parte, se non nel buio più fitto di un capannone sul porto, lasciandosi alle spalle l'ultima luce dell'edificio dei doganieri ("Oh l'orizzonte in fuga, dove s'accende/rara la luce della petroliera!", ricordava Montale proprio nella sua Casa dei doganieri).

Dunque Spino, l'anatomopatologo che si improvvisa investigatore cercando la vera identità del defunto Carlo Nobodi, non cercherebbe altri che se stesso. Ma questa suggestiva metafora esistenziale, metafisica o ermeneutica sottesa a tanti esempi di investigazione poliziesca (il giallo come ricerca dell'io o di Dio, dell'autore o del lettore), nel film non è solo suggerita, trova quasi un sugello (fanta)scientifico: le impronte digitali del protagonista, che muore in una sequenza identica a quella di apertura, risultano uguali a quelle del primo cadavere. Al contrario del romanzo, lo schermo aveva mostrato l'assassinio iniziale; e l'aveva mostrato per poi ripeterlo identico nei minuti finali. Sarà solo una suggestione dovuta al nome del regista, ma vengono in mente le ripetizioni stranianti di Un'ape sotto la pioggia. Lì servivano a destrutturare un romanzo costruito classicamente, con tanto di climax e di scioglimento finale; qui chiudono classicamente (in portoghese si direbbe rematam, che è un po' come dire "riammazzano": terminare è, appunto, uccidere due volte), in nome di una narratività ritrovata anche a fatica, un romanzo volutamente monco. L'adattamento

5 A. Tabucchi, Il filo dell'orizzonte, Milano, Feltrinelli, 1986 (le citazioni sono tratte dall'edizione del 1998, p. 107) 
per Fernando Lopes si rivela dunque una dialettica della contraddizione. Adattare diviene un sottrarsi a priori all'orizzonte d'attesa del fruitore ideale, in questo caso lo spettatore/lettore ("cette fameuse infidélité..." di certo cinema d'autore). Aveva ragione Fabio Ferzetti a ipotizzare che "uno studioso di Pessoa, scrittore dalle molte identità, non chiede altro che di essere tradito", ${ }^{6}$ ma l'effetto più paradossale (forse solo in apparenza?) è che il film risulta più astratto del libro. Tabucchi stesso, o chi per lui, in una lettera anonima/apocrifa scritta al regista alcuni anni dopo, dice:

[...] di quel romanzo di Tabucchi Lei ha saputo fare quello che Mondrian riuscì a fare con i rami intricati di un albero, trasformandoli in linee orizzontali e verticali. In questa Sua geometrizzazione, forse un po' intellettualistica, ma che riesce a quadrare il cerchio, ha comunque ristabilito una profonda verità: nel film il protagonista, Spino, è la stessa persona del cadavere che cerca $[\ldots] .^{7}$

Altro paradosso è che l'astrattezza (la quadratura alla Mondrian) viene raggiunta concretizzando. Oltre che con il tempo, manipolato in sala di montaggio (passione che abbiamo riconosciuto già nel cineasta degli esordi), c'è infatti da regolare i conti con lo spazio. La messa in quadro del libro esige che si dia un orizzonte reale alla città immaginaria che il romanziere non cita mai, che sappiamo essere simile a Genova, ma non disdegna, mascherati o tradotti, toponimi lisbonesi, pisani o semplicemente marittimi (Piazza delle Vettovaglie, Piazza del Parlasolo, Vico dei Calafati...).

${ }^{6}$ Nella recensione del film Rebus, di Massimo Guglielmi, in Il Messaggero, 18-02-1989, ora anche in http://www.cinematografo.it/pls/cinematografo/ consultazione.redirect?sch $=25877$.

7 A. Tabucchi, Autobiografie altrui. Poetiche a posteriori, Milano, Feltrinelli, 2003 , p. 62 , ove si riproduce una lettera che Lopes avrebbe ricevuto da uno spettatore del suo film. 
Quella città, nel film, diventa Lisbona. Una Lisbona prevalentemente notturna, poco "ville blanche", mai da cartolina, eppure sempre riconoscibile, ripresa in interni austeri o nei suoi quartieri a ridosso del fiume. Ma ciò che nel film si guadagna in geografia e topografia urbana (l'immersione in una città reale) si perde al livello del rapporto sempre in bilico (gioco in Tabucchi differito ma mai abbandonato) fra Storia e racconto, fra narrativa come sistema chiuso e nel contempo aperto al contingente e alla contemporaneità. Difatti il lettore italiano sa, o quantomeno si sente autorizzato a intuire, che quella probabile Genova del libro potrebbe essere la città dei conflitti a fuoco tra brigate rosse e forze dell'ordine a cavallo fra gli anni '70 e ' 80 , la città dei primi covi scoperti, delle prime colonne $\mathrm{Br}$ sgominate. Città di omicidi spietati e massacri cruenti, di tanti proiettili e cadaveri vaganti. Città in cui l'identificazione dei terroristi, innocui o addirittura cordiali inquilini della porta accanto, stimolò l'autoanalisi di buona parte della sinistra italiana ${ }^{8}$. Ne Il filo dell'orizzonte basterebbe il non detto, ma le reticenze del romanzo si completano con le letture parallele, a cominciare proprio dal biografismo della citata lettera a Fernando Lopes (auto o eterobiografica che essa sia), per poi passare ad altri racconti tabucchiani sul tema del fuggiasco e del ricercato: perché è morto il corpo senza nome de Il filo dell'orizzonte? Forse per lo stesso motivo per cui fugge il

8 Ed è difficile scacciare dalla mente un'affinità forse solo casuale: anche quell'autoanalisi in seno alla sinistra italiana era iniziata con una metafora fotografica: l' "album di famiglia” di un noto articolo firmato da Rossana Rossanda all'indomani del rapimento Moro. Metafora che nel romanzo di Tabucchi assume evidentemente un ruolo centrale: dal ritratto del cadavere sul giornale che, dice a Spino la sua compagna: "Con la barba e venti anni di meno potresti essere tu" (p. 32), fino alla foto trovata nel taschino del morto e stampata all'ingranditore nel capitolo 10, con quel gruppo di famiglia che "si rifiutava di tornare a esibirsi sul palco delle immagini per soddisfare la curiosità di una persona estranea, in un luogo estraneo, in un tempo che non è più il suo" (pp. 54-55). 
nome senza corpo di Notturno indiano, che fra l'altro condivide col narratore una certa simpatia per la canzone napoletana, come d'altronde giudice e imputato in Piccoli equivoci senza importanza, uno dei racconti in cui è più manifesto l'intento di tracciare il ritratto di una generazione, la stessa dell'autore, quella che è cresciuta nel dopoguerra, ha fatto il '68 e si è sparpagliata in una pluralità di scelte di segno ora più o meno coerente, ora diametralmente opposto agli slanci giovanili. Dunque, con agganci sempre più espliciti alla realtà persino cronachistica, in particolare dopo l'esplosione in Italia del caso Sofri, abbiamo visto Tabucchi entrare nella schiera degli intellettuali più combattivi, non solo in veste di panflettista ma anche di narratore, per così dire, "puro". Come ci ricorda in epigrafe La gastrite di Platone, a un racconto di Tabucchi spettò persino l'onore e l'onere di una citazione nella sentenza del $1 .^{\circ}$ processo d'appello contro Sofri, Bompressi e Pietrostefani, accusati, com'è noto, di un omicidio realizzato per vendicare un altro omicidio impunito, scaturito a sua volta dalla mendace pista d'indagine su una strage terroristica. La storia d'Italia, anche la più recente, non lesina catene (e caterve) di cadaveri con conseguente materiale narrativo. Il problema non è tanto la chiave per raccontarlo, quanto la motivazione per farlo.

Se è giusta l'ipotesi di Antonio Scurati, secondo cui la generazione della Resistenza, dei Calvino e dei Fenoglio, ha goduto per ultima della possibilità storica di saldare letteratura ed esperienza:

la possibilità che le letture e le esperienze di vita non siano due universi, ma uno, è esattamente questa la chance negata al nostro presente dal nuovo perverso rapporto stabilitosi tra realtà e immaginazione $[\ldots]^{9}$

9 Antonio Scurati, La letteratura dell'inesperienza. Scrivere romanzi al tempo della televisione, Milano, Bompiani, 2006, p. 30. 
Questo Tabucchi potrebbe essere visto come il primo "scrittore dell'inesperienza" che problematizza il rapporto fra realtà, memoria ed immaginazione lavorando sul materiale "bellico" della sua generazione: quella guerra a "bassa intensità" vissuta negli anni della guerra fredda e che pare destinata a prolungarsi negli anni del "dopo 11 settembre", in una società sempre più pervasa dai massmedia, i quali cambiano non solo il modo di percepire la guerra ma anche di farla; non solo il modo di fruire il dolore altrui ma anche il modo di infliggerlo. Se, sempre secondo Scurati, questa esperienza del dolore ci è stata sequestrata tramite la medicalizzazione segregante e la mediatizzazione dei conflitti, risulta ancor più significativo che sia un anatomopatologo, ossia quella pasta di medico più assuefatta alla frequentazione dei cadaveri, a decidere d'un tratto che quel morto smetterà di essere appena uno dei tanti, cercando di estrarlo dalla catena anonima dei decessi e dalla massa informe delle sciagure mondiali che sin dal giorno dopo invadono la redazione dove lavora l'amico giornalista, già distratto da Asia e Africa.

"Ma chi è lui per te?"10 chiede il pianista Harpo a Spino, detective anomalo che non cerca un assassino bensì il nome dell'assassinato; e chi è Ecuba per il teatrante? Scrive sempre Spino su un biglietto nel penultimo capitolo, dove si denuncia "la nostra impossibilità di cogliere i veri nessi delle cose che sono" "11. E proprio questa impossibilità di cogliere i nessi, questa rischiosa confusione tra immaginazione e memoria ("Che cosa sta inventando la sua immaginazione che si spaccia per memoria?"12 dice il narratore quando Spino osserva all'ingranditore la foto ritrovata) rischiano di far scivolare il racconto dell'esperienza nel delirio, parola chiave, insieme a "sogno", che da Pessoa si travasa nell'universo narrativo

\footnotetext{
10 A. Tabucchi, Il filo dell'orizzonte, p. 80.

11 Ib., p. 98.

12 Ib., p. 57-58.
} 
di Tabucchi, ma va a mescolarsi dialetticamente con altre parole chiave per l'autore di Piazza d'Italia e Sostiene Pereira: "memoria" e "coscienza". Per Scurati il delirio troverebbe il suo luogo ideale nel fictual:

nella regione paludosa dell'immaginario collettivo dove la distinzione tra il finzionale (fictional) e il fattuale (factual) non è più rilevante, prima ancora di non essere possibile, le trame nere che scrissero la nostra storia tragica si confondono alle trame oscure delle finzioni in serie. ${ }^{13}$

Perciò il finale (fanta)scientifico del film, a prima vista una di quelle situazioni che di solito aprono la narrazione, stavolta, come in una sceneggiatura rovesciata, si ferma un passo al di qua del mistero. E scivola nell'esoterico, laddove in Tabucchi trovavamo ancora un equilibrio difficile, giocato sulla continua e proficua possibilità di slittamento fra il giallo e la confessione, fra Genova e una qualunque città di Dio o enigmatico Nowhere, fra Nobodi e Nobody, fra Spino e Spinoza, fra il generale Dalla Chiesa e il commissario Calabresi, insomma fra il plot poliziesco e quello esistenziale ${ }^{14}$, il tutto ottenuto ora utilizzando e ora deponendo gli arnesi della letteratura di genere. Come ben sintetizza Jonathan Smith, Tabucchi:

[...] si trova costretto a raggiungere la politica attraverso il postmoderno, che è come dire raggiungere il reale attraverso l'apparenza. O, a rigor di termini, attraverso le apparenze. ${ }^{15}$

13 A. Scurati, op. cit., p. 24.

${ }^{14}$ Convivenza di plot paralleli ricordata da R. Ceserani, "Il filo dell'orizzonte: is it Luino or Duino the place to go?", in AA.VV. Antonio Tabucchi, geografia de um escritor inquieto, Lisboa, FCG, 2001.

15 L. Paci, Leggere Tabucchi; conversazione con Jonathan Smith, http:// www.swan.ac.uk/italian/movimento/mov1/archivio/I;\%204/tabucchi. html 
Al contrario Fernando Lopes, cineasta cinefilo, amante del noir e del polar francesi, nel suo trasloco lisbonese (via Parigi ${ }^{16}$ ) di quella stessa storia, sembra essersi domandato, in un tono più simile ad Amleto che a Paolo Conte, cosa fosse Genova per lui e lui per Genova. E, proprio come al tempo di Un'ape sotto la pioggia, ne ha ricavato un film il cui linguaggio funziona più come metalinguaggio. Un film che si interroga molto sul fare cinema, meno sul ruolo del cinema fuori dal cinema. Un film che, in direzione contraria a Tabucchi, raggiunge l'apparenza attraverso il reale che pure esibisce (proprio come fanno quei massmedia da cui certo cinema amerebbe distinguersi). Ennesima variante - fra scrittura e immagine, fra Italia e Portogallo, fra soggetti e discorsi distanti - di un altro gioco del rovescio.

16 Mercé gli sceneggiatori Jean Nachbaur e Christopher Frank. In particolare quest'ultimo, già collaboratore di Michel Deville e Alain Delon, adattatore di Colette e Patricia Higsmith, portava solido mestiere e una sufficiente "souplesse d'esprit". 